\title{
APLICAÇÃO DO MÉTODO SIMULATED ANNEALING PARA DETERMINAR AS ROTAS DE UMA EMPRESA DISTRIBUIDORA DE BEBIDAS
}

\author{
Josyane Negri Yamanaka da Silva \\ Centro Universitário do Leste de Minas Gerais (UNILESTE) \\ Av. Tancredo Neves, 3500, Bairro Universitário, CEP 35170-056, Cel. Fabriciano/MG \\ josyane.negri@hotmail.com

\begin{abstract}
Aloísio de Castro Gomes Júnior
Universidade Federal de Ouro Preto (UFOP)

Escola de Minas/DEPRO, Campus do Morro do Cruzeiro, CEP 35400-000, Ouro Preto/MG aloisio.junior@ufop.edu.br

Mateus Carlos da Silva

Centro Universitário do Leste de Minas Gerais (UNILESTE)

Av. Tancredo Neves, 3500, Bairro Universitário, CEP 35170-056, Cel. Fabriciano/MG mateus.m@outlook.com

Helton Cristiano Gomes

Universidade Federal de Ouro Preto (UFOP)

Escola de Minas/DEPRO, Campus do Morro do Cruzeiro, CEP 35400-000, Ouro Preto/MG helton.gomes@ufop.edu.br
\end{abstract}

\section{RESUMO}

O presente trabalho apresenta a aplicação da meta-heurística Simulated Annealing para a determinação das rotas dos veículos de uma empresa distribuidora de bebidas. $\mathrm{O}$ método Simulated Annealing foi escolhido por apresentar boas soluções para problemas de roteamento de veículos e ser de fácil implementação. O método foi desenvolvido em linguagem $\mathrm{C}$, usando-se o software $\mathrm{DEV} \mathrm{C++}$, e procura encontrar as rotas que minimizam as distâncias percorridas pelos veículos, procurando respeitar as restrições de capacidade dos veículos e das janelas de atendimento dos clientes. Os veículos usados pela empresa estudada possuem capacidades diferentes. Dessa forma, o problema aqui resolvido é caracterizado com um problema de roteamento de veículos com frota heterogênea e janela de tempo. Utilizou-se dados referentes a um dia de trabalho para três importantes cidades da região Leste do estado de Minas Gerais. Atualmente, as rotas na empresa estudada são definidas por um software denominado PathFind. Os resultados obtidos pelo método proposto se mostraram promissores, gerando soluções melhores que a solução usada pela empresa. No melhor caso, a melhora foi de 38,8\%.

Palavra-chave: Logística; Distribuição; Bebidas; Meta-heurísticas; Simulated Annealing.

\section{ABSTRACT}


This paper presents the application of the Simulated Annealing meta-heuristic for the determination of the routes of the vehicles of a beverage distribution company. The Simulated annealing method has been chosen for presenting good solutions for Vehicle Routing Problems and being easy to implement. The method was developed in $\mathrm{C}$ language, using the $\mathrm{DEV} \mathrm{C}++$ software, and seeks to find the routes that minimize the distances traveled by the vehicles, seeking to respect the constraints of vehicle capacity and customer service windows. The vehicles used by the studied company have different capacities. In this way, the problem solved here is characterized with a Vehicle Routing Problem with heterogeneous fleet and time window. Data referring to one day of work were used for three important cities in the eastern region of the state of Minas Gerais. Currently, the routes in the company studied are defined by a software called PathFind. The results obtained by the proposed method proved promising, generating better solutions than the solution used by the company. In the best case, the improvement was $38.8 \%$.

Keywords: Logistic; Distribution; Beverages; Meta-heuristics; Simulated Annealing.

\section{Como Citar:}

SILVA, Josyane Negri Yamanaka da; GOMES JÚNIOR, Aloísio de Castro; SILVA, Mateus Carlos da; GOMES, Helton Cristiano. Aplicação do Método Simulated Annealing para Determinar as Rotas de uma Empresa Distribuidora de Bebidas. In: XIX SIMPÓSIO DE PESQUISA OPERACIONAL E LOGÍSTICA DA MARINHA, 2019, Rio de Janeiro, RJ. Anais [...]. Rio de Janeiro: Centro de Análises de Sistemas Navais, 2019.

\section{INTRODUÇÃO}

A economia globalizada permitiu a eliminação das fronteiras territoriais viabilizando maior integração do comércio. À vista disto, a abertura dos mercados fez com que os clientes, cada vez mais exigentes, impulsionassem as empresas a oferecerem serviços e produtos com maior qualidade e menor prazo de entrega (COTA et al., 2017). Este cenário passou a exigir das organizações uma eficiente operação logística e, consequentemente, o desenvolvimento de estratégias nesta área.

Uma das estratégias logísticas a ser adotada é a busca pela redução de custos, que, segundo Ballou (2006), consiste em uma estratégia voltada para o enxugamento dos custos variáveis relacionados ao transporte e armazenagem. A minimização dos custos é um fator competitivo de suma importância para empresas que pretendem sobreviver e crescer no mercado atual. Em razão disso, os custos com transportes têm obtido mais visibilidade nas últimas décadas. Uma das formas de alcançar a minimização destes custos, é por meio da resolução do problema de roteamento de veículos (PRV). O PRV é um dos problemas de distribuição logística mais conhecido e estudado na área de otimização combinatória em virtude da sua aplicabilidade e importância, especialmente na cadeia de suprimentos (KRAMER et al., 2016).

A partir de um conjunto de clientes atendidos por meio de um determinado número de veículos, que partem dos depósitos (ou origens), o PRV consiste em encontrar as melhores rotas, tendo como um dos principais resultados a redução nos custos de transportes.

Nessa circunstância, o presente trabalho busca, por meio de um estudo no setor logístico de uma empresa situada na região Leste do estado de Minas Gerais, responder o seguinte questionamento: de que forma é possível reduzir os custos de entrega de uma empresa utilizando ferramentas de otimização? A fim de responder esse questionamento, utilizou-se a aplicação de uma metodologia baseada na meta-heurística Simulated Annealing para a determinação das melhores rotas para os veículos dessa empresa. O uso desse método deu-se pelo fato da complexidade do problema em questão, uma vez que os métodos exatos 
não conseguiram resolver problema com grande número de localidades a serem visitadas em um tempo computacional satisfatório.

Posto isso, o objetivo deste trabalho foi determinar o roteamento dos veículos por meio da definição das rotas de entrega de forma a minimizar os custos logísticos da empresa, a qual atua no ramo de distribuição de bebidas. Para isso, considerou-se apenas as rotas de algumas das principais cidades do Leste de Minas Gerais, clientes dessa empresa.

A relevância deste estudo relaciona-se com o fato de que a movimentação de cargas absorve de um a dois terços dos custos logísticos totais e por isso o operador logístico precisa ser um grande conhecedor da questão de transportes (BALLOU, 2006).

\section{REVISÃO BIBLIOGRÁFICA}

\subsection{Logística E CADEIA de Suprimentos}

$\mathrm{Na}$ área empresarial, acredita-se que a logística tem uma função limitada que envolve apenas atividades relacionadas ao transporte e armazenamento de mercadorias (DIAS, 2012). Porém, o autor acrescenta que, atualmente, a logística acompanha toda a movimentação de materiais e equipamentos da empresa, coordenando a compra, a movimentação, a armazenagem, o transporte e a distribuição física. Em uma visão mais abrangente, a logística empresarial compreende o acompanhamento do fluxo dos produtos, desde quando são consideradas matérias primas até o momento onde se faz seu descarte. Além de lidar com bens físicos, a logística também trabalha e acompanha o fluxo de serviços (BALLOU, 2006).

Bowersox et al. (2006) afirmam que, durante a década de 90, o mundo do comércio sofreu o impacto irrevogável dos avanços da informática, da internet e de uma série de possibilidades acessíveis de transmissão de informações. Os autores ainda explicam que a fácil disponibilidade de informações gerou grandes mudanças no mundo empresarial, onde a internet identificada por sua alta velocidade possibilitou a existência de um meio econômico mais veloz para conduzir transações e dessa maneira uma economia globalizada emergiu rapidamente. Dias (2012) complementa dizendo que, nos últimos anos, a globalização colocou a logística em um novo patamar.

Devido ao grande aumento da circulação de mercadorias, a pressão para reduzir custos e aumentar vendas, as empresas atentaram-se para a importância de desenvolver uma cadeia de suprimentos eficiente. A partir desta nova realidade, originou-se uma nova categoria de relacionamento entre negócios e foi chamada de gestão da cadeia de suprimentos - SCM (do inglês Supply Chain Management). A cadeia de suprimentos integra não só as áreas funcionais da empresa, como também a coordenação e o alinhamento dos esforços dos diversos departamentos (vendas, produção, financeiro e marketing) na busca de reduzir custos e agregar o máximo de valor ao cliente final. (DIAS, 2012)

A SCM é todo o esforço envolvido nos processos empresariais que acabam criando valor na forma de um produto ou determinado serviço para o consumidor final (SANTANA, 2016).

Para Ballou (2006), um conceito geral determina que as atividades empresariais criam quatro tipos de valores em produtos ou serviços, que são: forma, tempo, lugar e posse. O valor forma é gerado pela produção, pela fábrica; os valores tempo e lugar são controlados pela logística, respectivamente, pelo estoque e pelo transporte; o valor posse é gerado pelo marketing e pelas finanças, que facilitam a transferência da posse para o consumidor (ROSA, 2011).

A Figura 1 ilustra a divisão desses valores. 


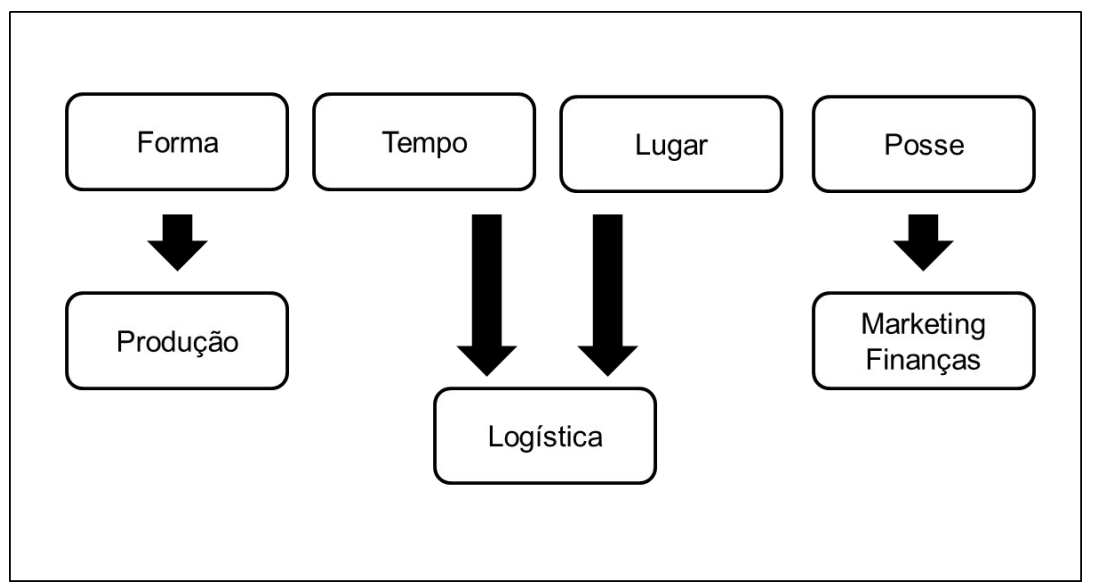

Figura 1: Valores que podem ser agregados ao produto

Fonte: Rosa (2011)

De acordo com a Figura 1, é possível observar que a logística controla metade dos processos que agregam valor a um produto. Desta forma, o valor gerado pela operação logística pode ser expresso de duas formas: tempo e lugar.

Ballou (2006) afirma que produtos e serviços não têm valor a menos que estejam em poder dos clientes quando (tempo) e onde (lugar) eles pretenderem consumi-los. Segundo Hara (2011), as utilidades de tempo e local são fornecidas pelo transporte. Embora as decisões sobre transportes se manifestem automaticamente em uma variedade de formatos, as principais são: a seleção do modal, a roteirização dos embarques, a programação dos veículos e a consolidação dos fretes (BALLOU, 2006).

Este trabalho tem seu foco na roteirização ou roteamento de veículos, conforme descrito na seção a seguir.

\subsection{RoteAmento de Veículos}

A decisão de uma empresa de como definir a melhor maneira de utilização de seus recursos é uma importante questão. Ragsdale (2010) diz que, no ambiente comercial competitivo de hoje, é cada vez mais importante garantir que os recursos limitados de uma empresa sejam usados da maneira mais eficiente possível. Dentro da área de transportes, um dos recursos que devem ser bem aproveitados e utilizados são os veículos que realizam as entregas das organizações.

Os problemas de roteamento de veículos (PRV) estão entre os mais complexos da área de otimização combinatória. O PRV busca estabelecer um conjunto de rotas ótimas para veículos, sendo que, cada uma dessas possui início e fim no depósito.

Belfiore et al. (2006), explicam que os PRV's têm recebido muita atenção nos últimos anos em função de sua aplicabilidade e importância econômica na determinação de estratégias eficientes de distribuição, com o objetivo de reduzir os custos operacionais no sistema de distribuição.

De acordo com Arenales et al. (2007), este tipo de problema tem um papel fundamental na área de gerenciamento da distribuição e logística, por isso é necessária a garantia de que as rotas dos veículos sejam definidas com o menor custo possível e com a máxima eficiência. Em suma, segundo Goldbarg e Luna (2005), a ideia básica do problema de roteamento é a determinação de sequências de visitas que objetivem atender a uma determinada função objetivo.

Os PRV's podem ser representados por grafos onde os nós representam os clientes que deverão ser atendidos e o depósito de onde partem as entregas. A Figura 2 apresenta um exemplo de grafo, onde o número 0 representa o depósito e os demais números os clientes. 


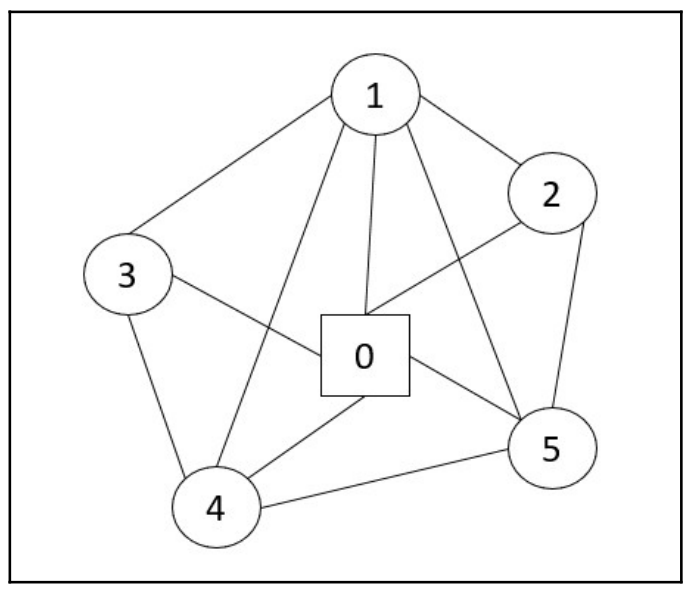

Figura 2: Exemplo de grafo

O PRV com frota heterogênea (PRVFH) é uma variação do PRV clássico, onde os veículos possuem capacidades distintas. Nesta variante, duas situações podem ocorrer: quando o número de veículos de cada tipo é conhecido, a frota é limitada, caso contrário, é considerada ilimitada. Neste último caso, além dos roteiros e da configuração ideal dos veículos em termos de tamanho, é necessário determinar também a composição da frota (BELFIORE et al., 2006; KRAMER et al., 2016).

Belfiore et al. (2006) dizem que objetivo do PRVFH é minimizar a soma dos custos fixos dos veículos e dos custos variáveis de roteirização. No entanto, Kramer et al. (2016) explicam que os custos fixos e/ou variáveis associados a cada tipo de veículo podem ou não ser considerados na modelagem.

Outra variante do PRV, é o problema de roteamento de veículos com janela de tempo (PRVJT). No PRVJT, além das restrições do PRV tradicional inclui-se o atendimento dos clientes dentro de um intervalo de tempo, determinado pelo cliente, também chamado de janela de tempo.

Um modelo matemático para a resolução do PRVJT é apresentado por Arenales et al. (2007). A seguir é apresentado esse modelo adaptado ao PRVJT com frota heterogênea.

As variáveis de decisão para o problema são:

- $\quad x_{i j k} \equiv\left\{\begin{array}{l}1, \text { se o veículo } k \text { faz uma viagem da cidade } i \text { para a cidade } j \\ 0, \text { caso contrário }\end{array}\right.$

- $s_{i k} \equiv$ instante em que o veículo ${ }^{k}$ começa a servir o cliente ${ }^{i}$.

E os dados do problema são:

- $n \equiv$ número de clientes a serem atendidos;

- $m \equiv$ número de veículos;

- $d_{i} \equiv$ demanda do cliente ${ }^{i}$;

- $\quad c_{i j} \equiv$ distância entre a cidade ${ }^{i}$ e a cidade $j$;

- $t_{i j} \equiv$ tempo de viagem entre a cidade ${ }^{i}$ e a cidade $i$;

- $t s_{i} \equiv$ tempo de serviço para atender o cliente ${ }^{i}$; 
- $Q^{k} \equiv$ capacidade do veículo $k$;

- $a_{i} \equiv$ início da janela de atendimento do cliente ${ }^{i}$.

- $b_{i} \equiv$ fim da janela de atendimento do cliente $i$.

- $C \equiv$ conjunto de cidades;

- $\quad N \equiv$ conjunto de cidades incluindo o depósito $\left({ }^{0}, n+1\right)$;

- $V \equiv$ conjunto de veículos.

- $E \equiv$ conjunto de possíveis conexões entre os clientes.

Obs.: Para fins de modelagem, neste problema todos veículos devem partir de ${ }^{0} \mathrm{e}$ chegar em $n+1$, ambos representando o centro de distribuição (depósito).

E o modelo matemático é descrito pelas equações (1) a (10).

$\operatorname{Min} Z=\sum_{i \in N} \sum_{j \in N} \sum_{k \in V} c_{i j} x_{i j k}$

Sujeito à:

$\sum_{k \in V} \sum_{j \in N} x_{i j k}=1 \quad \forall i \in C$

$\sum_{i \in C} d_{i} \sum_{j \in N} x_{i j k} \leq Q^{k} \quad \forall k \in V$

$\sum_{j \in N} x_{0, j, k}=1 \quad \forall k \in V$

$\sum_{i \in N} x_{i, h, k}-\sum_{j \in N} x_{h, j, k}=0 \forall h \in C, \forall k \in V$

$\sum_{i \neq N} x_{i, n+1, k}=1 \quad \forall k \in V$

$s_{i k}+t s_{i}+t_{i j} \leq s_{i k}+\left(1-x_{i j k}\right) M \forall k \in V e \forall(i, j) \in E$

$a_{i} \leq s_{i k} \leq b_{i} \forall k \in V, \forall i \in N-\{0\}$

$x_{i, i, k} \in\{0,1\} \forall i \in N, \forall j \in N, \forall k \in V$

$s_{i k} \geq 0 \quad \forall i \in N, \forall k \in V$

A equação (1) procura minimizar os custos totais com transporte. As restrições (2) 
asseguram que cada cliente ${ }^{i}$ é designado a um único veículo $k$. As restrições (3) impõem que a demanda total de cada veículo ${ }^{k}$ não exceda a capacidade $Q^{k}$ do mesmo. As restrições (4) a (6) exigem que cada veículo parta uma única vez do depósito (nó ${ }^{0}$ ), deixe o nó ${ }^{h}$ só e somente se entrar neste nó e retorne ao depósito (nó $n+1$ ) uma única vez. As restrições (7) garantem que se o veículo ${ }^{k}$ deixa o nó ${ }^{i}$ e viaja para o nó $i$, então ele não pode chegar em $i$ antes de $s_{i k}+t s_{i}+t_{i i}$. As restrições (8) asseguram o período de entrega (janela de tempo) será respeitado. As restrições (9) e (10) definem o domínio das variáveis do problema.

Uma breve revisão literária à cerca da utilização do PRV é apresentada a seguir.

Gomes Jr. et al. (2005), com o objetivo de determinar rotas de custo mínimo para uma frota de veículos de mesma capacidade e atendendo as demandas dos clientes com períodos determinados, apresentou um algoritmo baseado na meta-heurística Simulated Annealing para resolver o PRV com janela de tempo (PRVJT) com frota homogênea. A metodologia foi aplicada a 168 problemas-teste da literatura e 13 novos melhores resultados foram encontrados.

O trabalho de Kramer et al. (2016) apresentou um estudo realizado em uma indústria de bebidas utilizando o PRV assimétrico com frota heterogênea limitada (PRVAFHL). O objetivo foi reduzir os custos de distribuição através da definição de melhores rotas para os veículos obedecendo as restrições de tempo máximo de duração de cada rota. Devido à alta complexidade computacional do problema, optou-se por fazer o uso do método heurístico baseado nas meta-heurísticas ILS e VND, que se mostrou eficiente ao obter uma solução com $14 \%$ de melhora em relação às soluções adotadas pela empresa, em termos de distância percorrida por cada veículo.

Leite e Gomes Júnior (2017) apresentaram um estudo realizado em uma empresa que contava com caminhões para distribuir seus produtos e realizar a operação de logística reversa simultaneamente. O objetivo foi avaliar se um modelo de programação linear inteira (PLI) que descreve o PRV com coleta e entrega simultânea (PRVCES) traria resultados mais satisfatórios que o software utilizado pela empresa, o qual era baseado na heurística do Vizinho Mais Próximo. Os resultados obtidos mostraram que o modelo de PLI trouxe uma redução de custo de $24,1 \%$ comparando-se à política de roteamento que era utilizada pela empresa.

\subsection{MÉtodos MetA-HEURÍsticos E O SimULATED ANNEALING}

De acordo com Hillier e Lieberman (2013), uma meta-heurística consiste em um método de resolução geral que fornece tanto uma estrutura quanto diretrizes de estratégias gerais para desenvolver um método heurístico específico, que ajuste-se a um tipo de problema particular. Contrariamente às heurísticas convencionais, as meta-heurísticas são de caráter geral e providas de mecanismos para evitar que a busca por soluções fique presa em ótimos locais possivelmente distantes dos ótimos globais. As meta-heurísticas diferenciamse entre si, basicamente, pelo mecanismo usado para sair das armadilhas dos ótimos locais. As principais meta-heurísticas encontradas na literatura são a Busca Tabu (BT), o Simulated Annealing (SA) e os Algoritmos Genéticos (AG), porém, mais recentemente, outros métodos têm sido utilizados, como o GRASP, o VNS e o ILS. Neste trabalho optou-se pela utilização do SA devido às inúmeras aplicações de sucesso do método na resolução de PRV's.

A meta-heurística SA, proposta por Kirkpatrick et al. (1983), é um método de busca local probabilístico que fundamenta-se em uma analogia com a termodinâmica, ao simular o resfriamento de um conjunto de átomos aquecidos, operação conhecida como recozimento. 
Um método de busca local realiza uma série de iterações, melhorando a solução de um determinado problema, através de movimentos dentro de uma estrutura de vizinhança, até chegar a um ótimo local. O SA possui uma estratégia para escapar de ótimos locais, aceitando soluções de piora com uma determinada probabilidade. A Figura 3 apresenta um pseudocódigo básico do SA, apresentado por Souza (2011), para um problema de minimização.

No pseudocódigo do da Figura 3, $\Delta$ é a variação no valor da função objetivo ao mover-se de uma solução s para uma solução s', isto é, $\Delta=\mathrm{f}\left(\mathrm{s}^{\prime}\right)-\mathrm{f}(\mathrm{s})$. O SA aceita o movimento e a solução s' passa a ser a solução corrente caso $\Delta<0$, ou seja, s' é melhor do que s. Caso $\Delta \geq 0$, a solução $s^{\prime}$ também pode ser aceita, mas neste caso, com uma probabilidade $e^{-\Delta / T}$, onde $\mathrm{T}$ é um parâmetro do método, denominado temperatura e que regula a probabilidade de aceitação ou não de uma solução de piora. A temperatura assume inicialmente um valor elevado $T_{0}$ e, após um número fixo de iterações ( $\left.S A_{\max }\right)$, é gradativamente reduzida por uma razão de resfriamento $\left({ }^{\alpha}\right)$. À medida que ${ }^{T}$ tende a zero, diminui-se a probabilidade de aceitação de soluções de piora. O procedimento para quando a temperatura aproxima-se de zero e nenhuma solução de piora é mais aceita, isto é, quando o sistema torna-se estável.

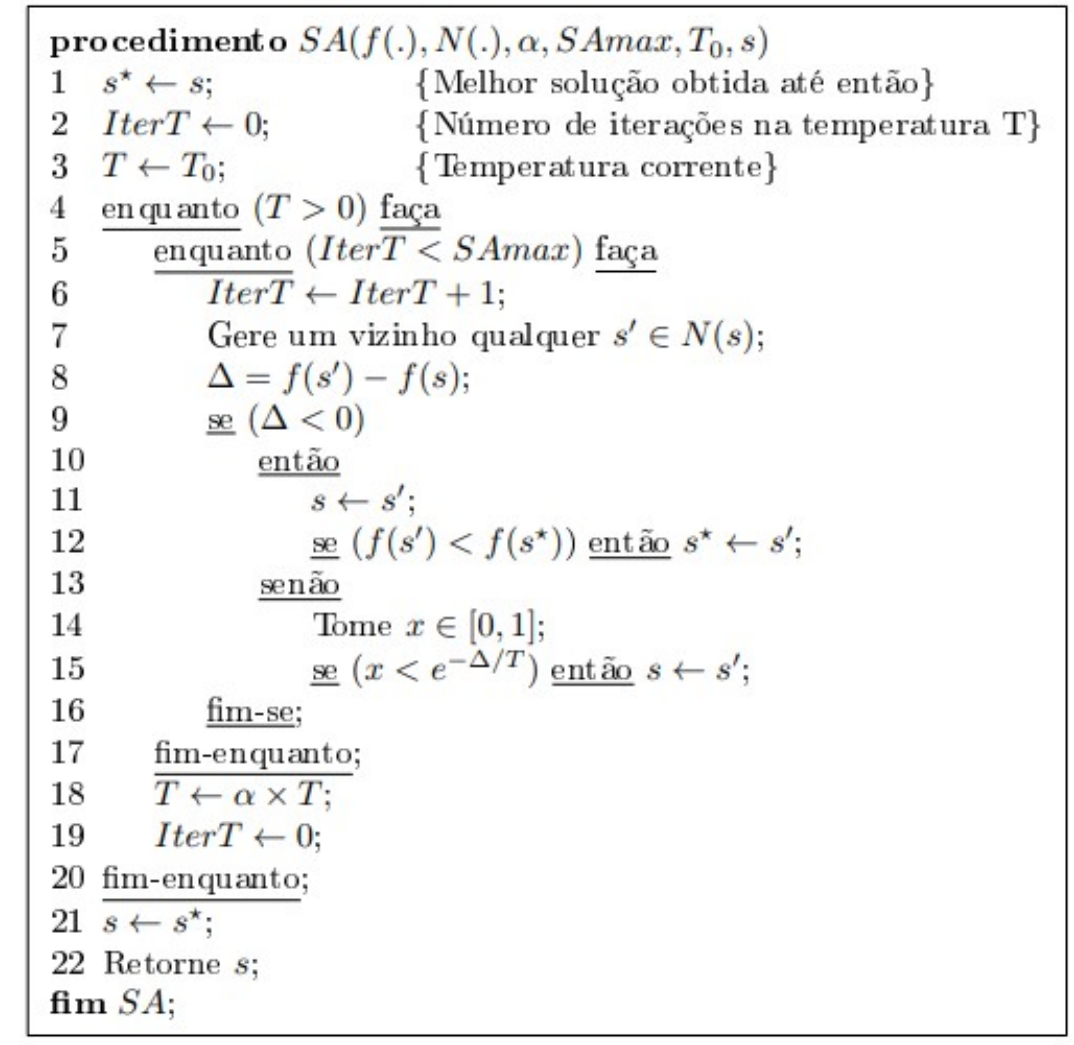

Figura 3: Pseudocódigo básico do SA aplicado a um problema de minimização

Fonte: Souza (2011).

Segundo Kirkpatrick et al. (1983), a velocidade de convergência para boas soluções do SA depende da definição de seus parâmetros. Os valores ideais para estes não podem ser determinados antecipadamente, eles dependem do problema específico que está sendo estudado e devem ser determinados através de testes estatísticos. 
Na seção a seguir é apresentado como o SA foi aplicado na resolução do PRV.

\section{SIMULATED ANNEALING APLICADO À RESOLUÇÃO DO PRVFHJT}

O método meta-heurístico SA utilizado neste trabalho para resolver o problema da empresa distribuidora de bebidas foi implementado na linguagem $\mathrm{C}$, utilizando o software Dev C++. O computador utilizado para a execução do programa foi um Intel Core i3 ${ }^{\mathrm{TM}}$ 2.00GHz, 4GB de memória RAM, sob sistema operacional Microsoft Windows 1064 bits.

\subsection{DESCRIÇÃO DO PROBLEMA}

Para o desenvolvimento do trabalho utilizou-se um cenário real de uma empresa distribuidora de bebidas localizada na região Leste do estado de Minas Gerais. A empresa em questão é uma sociedade empresarial limitada, que tem como atividades principais o comércio atacadista e distribuição de produtos. A pesquisa foi desenvolvida no setor logístico da empresa, analisando-se as tomadas de decisões relacionadas ao transporte, mais especificamente sobre o roteamento dos seus veículos de entregas. Para a sua elaboração, levou-se em consideração apenas os dados e informações referentes à matriz, que atende algumas das principais cidades do Leste de Minas Gerais entre outras cidades menores situadas nessa mesma região.

Após os pedidos de vendas serem coletados pelos vendedores, juntamente com seus respectivos clientes, eles são entregues ao setor logístico para que se possa determinar o esquema de entrega. Todos os veículos utilizados pela empresa são terceirizados, a qual tem contrato fixo de 5 caminhões/dia, independente da distância a ser percorrida. Por essa razão, o interesse da empresa é maximizar o uso dos caminhões disponíveis, para não haver necessidade de realizar pagamento de caminhão adicional. Os caminhões saem do centro de distribuição às 8 horas e devem retornar até no máximo as 18 horas, por conta de leis trabalhistas, sendo que devem encerrar suas atividades por volta das 17 horas para retornarem à empresa a tempo.

Atualmente o roteamento dos veículos é realizado por meio do software chamado PathFind, que de acordo com o gestor da empresa estudada, primeiramente agrupa os clientes conforme os bairros e em seguida constrói as rotas de acordo com as distâncias entre os clientes, de acordo com a localização mais próxima.

\subsection{METODOLOGIA PARA A RESOLUÇÃO DO PROBLEMA}

Para a elaboração do problema e posterior implementação do SA, primeiramente foi feita a coleta dos dados necessários, os quais foram fornecidos em planilhas pelo gestor responsável pela logística da empresa. As planilhas fornecidas continham dados relacionados ao período de um dia de trabalho, e compreendiam as rotas utilizadas para as entregas na região do Vale do Aço e regiões próximas. Desta forma, selecionaram-se informações necessárias, tais como: clientes atendidos; endereço dos clientes atendidos; demanda (em Kg) de cada cliente; quantidade de veículos utilizados; capacidade (em Kg) dos veículos utilizados; taxa de ocupação de cada veículo utilizado; e rotas que foram utilizadas.

Foi preciso a manipulação de alguns dados, pois os mesmos não se apresentavam na forma como seriam trabalhados. Nessa etapa foram calculadas, também, as distâncias entre os clientes utilizando a ferramenta Google Maps.

Para a realização desse trabalho foram consideradas apenas as principais cidades do Vale do Aço: Ipatinga, Coronel Fabriciano e Timóteo, as quais consistiam em uma totalidade de 161 clientes. E para a entrega nessa região a empresa conta com 4 veículos, sendo 1 
veículo com capacidade de $6820 \mathrm{Kg}$ e 3 veículos com capacidade de $6820 \mathrm{Kg}$. Além disso, foram utilizados os dados de um dia de entrega nessas três cidades.

O problema aqui apresentado pode ser considerado com um problema de roteamento de veículos com frota heterogênea e janela de tempo (PRVFHJT).

Nas seções seguintes são apresentados os elementos principais do método SA elaborado, a saber: (i) representação de uma solução; (ii) Estruturas de vizinhanças adotadas; (iii) geração de uma solução inicial; (iv) Função de avaliação; (v) Parâmetros do método SA. O método desenvolvido foi o apresentado na Figura 3.

\subsubsection{Representação de uma solução}

Uma solução para o PRVFHJT pode ser representada conforme a Figura 4. Na representação proposta na Figura 4, um veículo de capacidade 200 sai do centro de distribuição (CD), visita as localidades 100, 120 e 130, nesta ordem, e retorna ao CD. O segundo veículo, de capacidade 400, sai do CD, visita as localidades 1, 3, 5 e 9, nesta ordem, e retorna ao $\mathrm{CD}$.

A solução apresentada na Figura 4 é composta por duas rotas, sendo a primeira (Rota 1) realizada por um veículo e a segunda (Rota 1) por outro veículo. As duas rotas podem ou não ser realizadas simultaneamente.

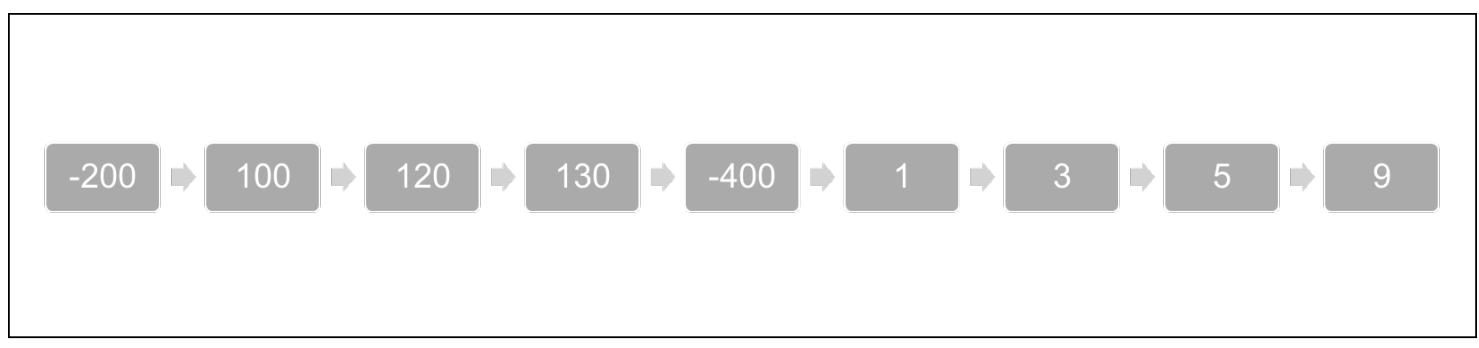

Figura 4: Exemplo de Representação de uma solução para o PRVFH

\subsubsection{Estrutura de Vizinhança}

Dada uma solução ${ }^{S}$, conforme a Figura 4, uma solução vizinha $S^{\prime}$ é obtida a partir de um movimento que consiste na simples troca de dois clientes distintos, que pode ser feita dentro da própria rota ou de uma rota para outra. As Figuras 5 e 6 representam como funciona as estruturas de vizinhança.

Na Figura 5, a ordem do cliente 84 é trocada, dentro da própria rota, com a do cliente 69. Já na Figura 6, a ordem do cliente 84, na Rota 1, é trocada com a do cliente 3, na Rota 2.

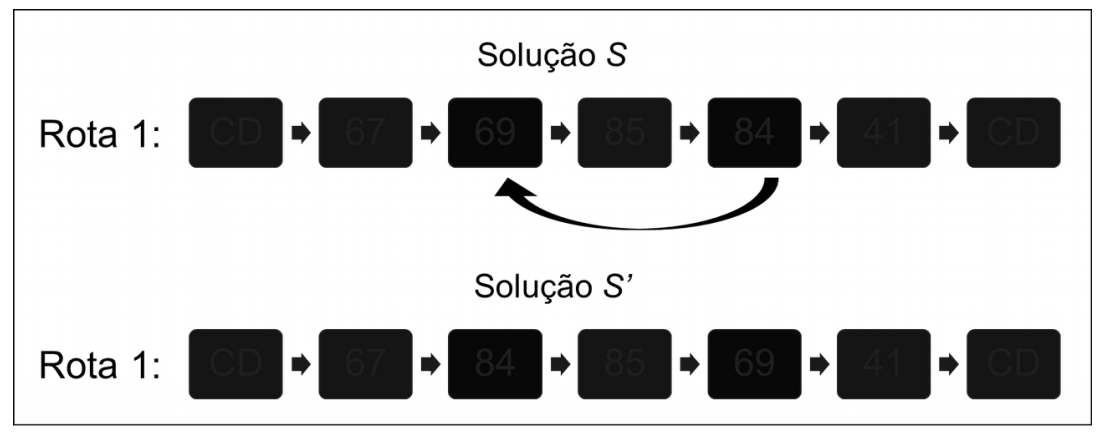

Figura 5: Troca dentro da própria rota 


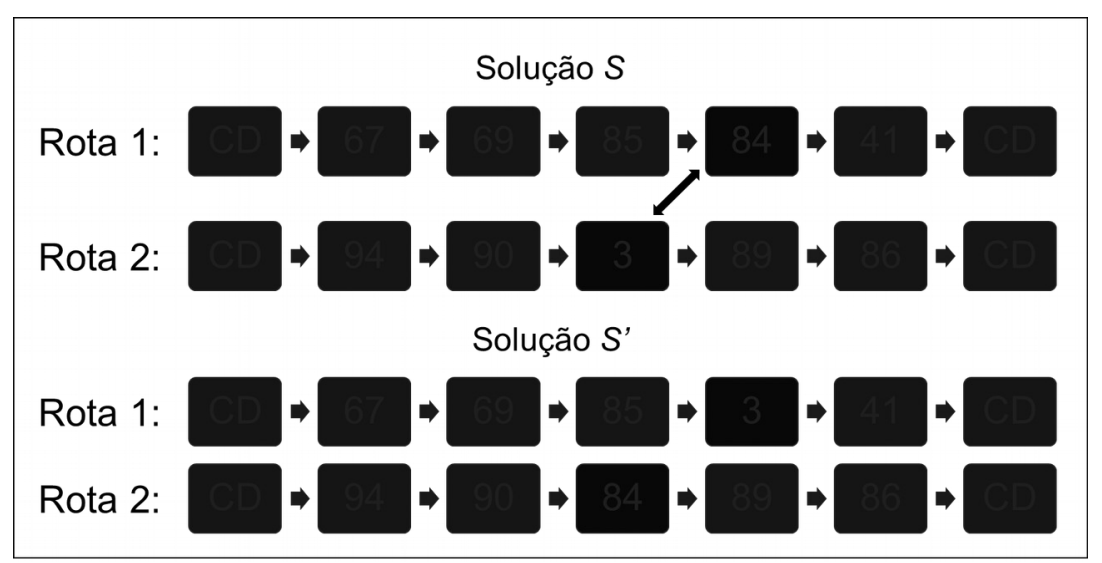

Figura 6: Troca em rotas diferentes

Para acontecer uma troca, escolhe-se um cliente aleatoriamente e troca-se a ordem com outro cliente qualquer.

\subsubsection{Função de Avaliação}

A função de avaliação utilizada, proposta por Gomes Júnior (2005), leva em consideração o custo de transporte ao realizar uma viagem do cliente i para outro cliente j, a capacidade do veículo que irá atender a rota e a janela de tempo em que os clientes devem ser atendidos. A função de avaliação é dada pela equação (11):

$$
f(x)=\sum_{\mathrm{i}=0}^{\mathrm{n}} \sum_{\mathrm{j}=0}^{\mathrm{n}} c_{i j} x_{i j}+\gamma f_{1}(x)+\beta f_{2}(x)
$$

Onde:

$c_{i j}=$ custo de transporte do cliente i para o cliente $\mathrm{j}$;

$f_{1}(x)=$ sobrecarga, ou seja, o excesso de peso nos veículos;

$f_{2}(x)=$ tempo excedido no horário máximo permitido para atendimento de um cliente;

$\gamma=$ penalidade para o excesso de carga nos veículos;

$\beta=$ penalidade para o tempo excedente ao final da janela de tempo de algum cliente.

No método desenvolvido, adotou-se $\gamma$ igual ao valor da maior distância (ou custo) entre dois clientes quaisquer vezes o número total de clientes e $\beta$ igual a 1000. Esses valores foram definidos para que soluções inviáveis tenham uma baixa probabilidade de serem escolhidas.

\subsubsection{Parâmetros para aplicação do Simulated Annealing}

Para uma utilização eficiente do SA é preciso definir seus parâmetros de controle, os quais serão utilizados na sua execução. No SA, os principais parâmetros são: a temperatura inicial $\left(T_{0}\right)$, o número máximo de iterações sem melhora ( $S A_{\max }$ ), taxa de 
resfriamento $\left({ }^{\alpha}\right)$ e a temperatura final $\left({ }^{T} f\right)$. Para determinar os valores desses parâmetros, foram testados diversos valores para os quatros parâmetros usando-se problemas encontrados na literatura e a combinação de valores que gerou os melhores resultados são apresentados na Tabela 1.

\begin{tabular}{|c|c|c|c|}
\hline Temperatura inicial $\left(T_{0}\right)$ & $S A_{\max }$ & Taxa de resfriamento $(\alpha)$ & Temperatura final \\
\hline 100000,0 & 5 & 0,998 & 0,001 \\
\hline
\end{tabular}

\subsection{Resultados e AnÁlise}

O método SA foi aplicado para gerar as rotas de entrega dos veículos, que possuem capacidades de carga diferentes. Por tratar-se de um método probabilístico, e possuir escolhas aleatórias, a cada execução do método, um novo conjunto de rotas é gerado. Sendo assim, o programa foi executado 20 vezes, originando 20 soluções diferentes, conforme a Tabela 2. Nessa tabela, a primeira coluna apresenta o número da solução gerada, a segunda coluna apresenta a solução encontrada (de acordo com a função de avaliação), a terceira coluna apresenta a distância total percorrida pelo veículo (em $\mathrm{Km}$ ), a quarta e a quinta colunas apresentam o tempo computacional dispendido para obter a solução (em segundos e em minutos, respectivamente) e a última coluna apresenta o tempo que foi excedido da janela de tempo (em minutos).

Conforme a Tabela 2, é possível observar que a melhor solução obtida foi a 11, apresentando a menor distância total percorrida, isto é, 221,5 Km. Em função disso, selecionou-se a mesma para realizar a comparação com a solução praticada pela empresa, por meio dos Km percorridos. Observa-se, também, que as soluções 9, 12 e 13 são inviáveis em relação à janela de atendimento, ou seja, não atenderam às jornadas de trabalho dos motoristas. Além disso, o tempo computacional médio para os 20 testes foi de 14,45 minutos. Outro fato que deve ser destacado é que todas as soluções viáveis geradas pelo SA foram melhores que a solução utilizada pela empresa. 


\begin{tabular}{cccccc}
\hline $\begin{array}{c}\text { Número da } \\
\text { solução }\end{array}$ & $\begin{array}{c}\text { Solução } \\
\text { encontrada }\end{array}$ & $\begin{array}{c}\text { Distância total } \\
\text { percorrida (em Km) }\end{array}$ & $\begin{array}{c}\text { Tempo } \\
\text { computacional (em } \\
\text { segundos) }\end{array}$ & $\begin{array}{c}\text { Tempo } \\
\text { computacional } \\
\text { (em min aprox.) }\end{array}$ & $\begin{array}{c}\text { Tempo que } \\
\text { passou da JT } \\
\text { total (em min) }\end{array}$ \\
\hline 1 & 236,5 & 236,5 & 838,9 & 14 & 0 \\
2 & 234,2 & 234,2 & 818,45 & 14 & 0 \\
3 & 224,9 & 224,9 & 833,4 & 14 & 0 \\
4 & 230,7 & 230,7 & 833,05 & 14 & 0 \\
5 & 239,3 & 239,3 & 850,74 & 14 & 0 \\
6 & 262,6 & 262,6 & 857,67 & 14 & 0 \\
7 & 239,5 & 239,5 & 934,83 & 16 & 0 \\
8 & 231,2 & 231,2 & 887,34 & 15 & 0 \\
9 & 1868007,75 & 302,6 & 835,14 & 14 & 146,1 \\
10 & 226,6 & 226,6 & 809 & 13 & 0 \\
11 & 221,5 & 221,5 & 822,83 & 14 & 0 \\
12 & 184382,38 & 272,8 & 842,68 & 14 & 148,2 \\
13 & 27385,5 & 685,5 & 884,84 & 15 & 26,7 \\
14 & 231,7 & 231,7 & 857,33 & 14 & 0 \\
15 & 254,7 & 254,7 & 885,91 & 15 & 0 \\
16 & 249,3 & 249,3 & 877,64 & 15 & 0 \\
17 & 232,6 & 232,6 & 899,16 & 15 & 0 \\
18 & 234,5 & 234,5 & 879,06 & 15 & 0 \\
19 & 241,7 & 241,7 & 884,37 & 15 & 0 \\
20 & 244,8 & 244,8 & 877,61 & 15 & 0 \\
\hline
\end{tabular}

Tabela 2: Soluções obtidas e suas respectivas informações

As rotas utilizadas pela empresa resultam em uma distância total de 362,4 Km, tendo um custo de $\mathrm{R} \$ 507,36$ (considerando-se apenas os custos com combustíveis e o custo do diesel de R \$ 4,09 por litro). A melhor solução obtida pelo SA apresentou uma distância total de 221,5 Km, com um custo de R \$ 310,10 (também considerando-se apenas os custos de combustíveis). Ao determinar um novo conjunto de rotas, os comparativos expostos apresentaram uma redução de 38,8\% nas distâncias percorridas e, consequentemente, a redução dos custos de entrega referentes ao período de 1 dia de trabalho. Sendo assim, podese afirmar que por meio da utilização de ferramentas de otimização é possível reduzir custos logísticos relacionados ao transporte de mercadorias.

A Figura 7 ilustra as rotas que foram realizadas pela empresa no período de 1 dia de trabalho, atendendo as principais cidades do Vale do Aço, e suas respectivas quilometragens. A Figura 8 apresenta as rotas geradas pelo método Simulated Annealing (melhor solução), atendendo os mesmos clientes, e suas respectivas quilometragens. 


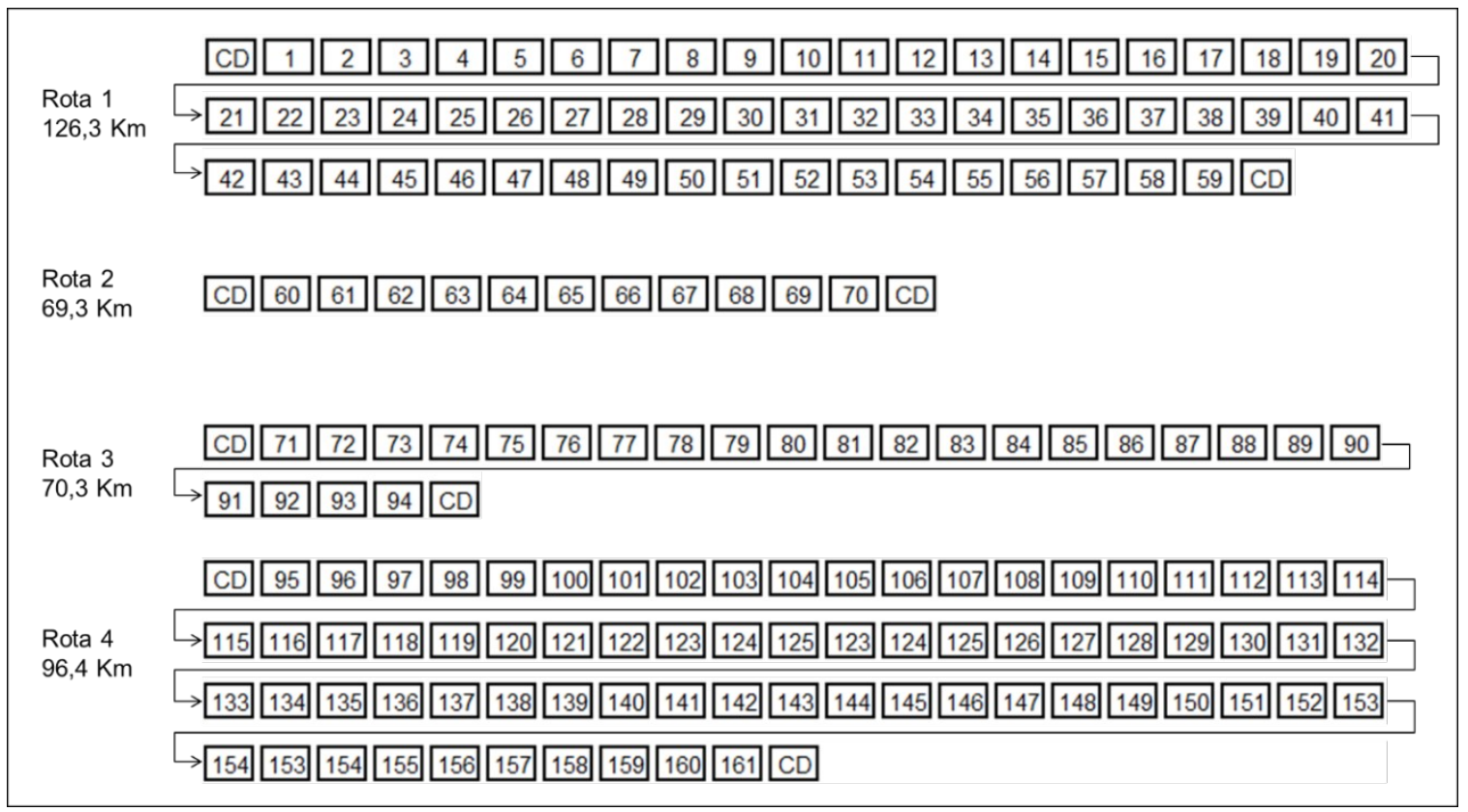

Figura 7: Rotas realizadas pela empresa

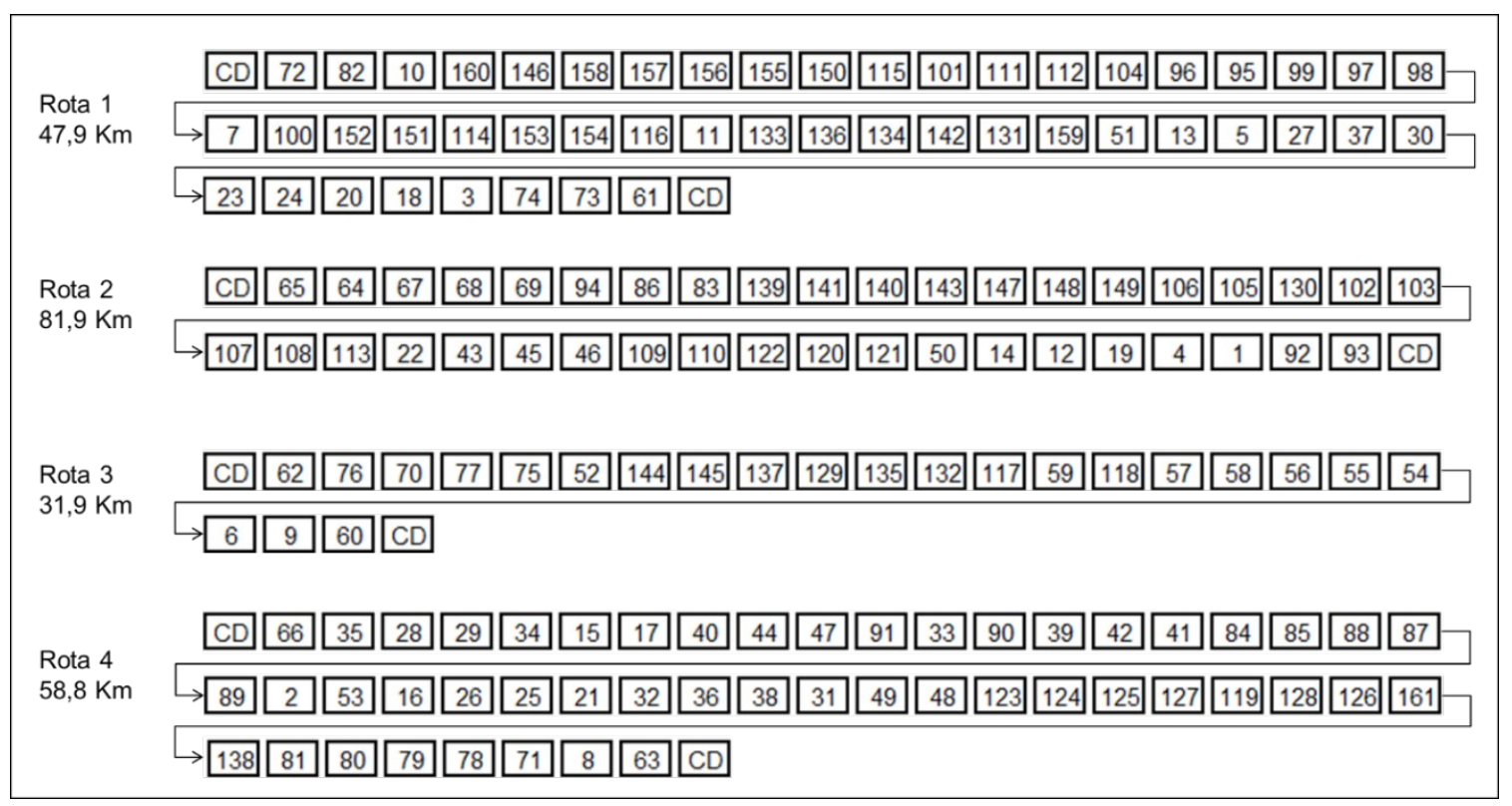

Figura 8: Rotas geradas pelo método SA

\section{CONSIDERAÇÕES FINAIS}

Este trabalho abordou o PRVFHJT e, propôs a utilização de um método de otimização para sua resolução, considerando dados de um problema real de uma empresa distribuidora de bebidas. Optou-se pela utilização do método Simulated Annealing, pois é uma técnica de fácil implementação e é capaz de gerar boas soluções.

A empresa estudada cedeu os dados referente ao período de 1 dia de trabalho em três importantes cidades da região Leste do estado de Minas Gerais para a elaboração desse trabalho. Essa empresa determinava as rotas de entrega de mercadorias por meio de um software chamado PathFind.

O objetivo proposto foi o de determinar o roteamento dos veículos da empresa, de 
forma a minimizar os custos logísticos relacionados ao transporte. Dos 20 testes realizados, 17 soluções geradas apresentam solução melhor comparando-se com a solução utilizada pela empresa. Dessa forma, pode-se perceber que, mesmo usando um software específico para gerar as rotas dos veículos, há possibilidades de melhoria, visto que os resultados obtidos pelo SA foram 38,8\% melhores do que a rota gerada pelo software da empresa estudada.

Como trabalhos futuros, sugere-se a inserção de demandas de coletas para os clientes, uma vez que em alguns momentos a empresa precisa recolher os engradados vazios. Além disso, pode-se testar outros métodos meta-heurísticos para gerar soluções para esse problema.

\section{REFERÊNCIAS BIBLIOGRÁFICAS}

[1] ARENAlES, M.; ARMENTANO, V.; MORABITO, R.; YANASSE, H. Pesquisa operacional. Rio de Janeiro: Elsevier, 2007.

[2] BALLOU, R. H. Gerenciamento da cadeia de suprimentos/logística empresarial. 5. ed. Porto Alegre: Bookman, 2006.

[3] BELFIORE, P.; FÁVERO, L. P. Pesquisa operacional: para cursos de engenharia. Rio de Janeiro: Elsevier, 2013.

[4] BELFIORE, P. P.; FÁVERO, L. P. L.; ALVAREZ, R. A. G. Problema de roteirização de veículos com frota heterogênea: revisão da literatura. In: Simpósio Brasileiro de Pesquisa Operacional, XXXVIII, 2006, Goiânia. Anais. Goiânia, 2006.

[5] BOWERSOX, D. J.; CLOSS, D. J.; COOPER, M. B. Gestão logística de cadeia de suprimentos. Porto Alegre: Bookman, 2006.

[6] COTA, B. M.; MARQUES, D. A. M.; CASTRO, F. J. F. de; GOMES JÚNIOR, A. C. Utilização da roteirização de veículos para redução do custo de transporte de funcionários e definição da escala de trabalho em uma empresa do ramo de transporte ferroviário. In: ENEGEP, XXXVII, 2017, Joinville. Anais. Joinville, 2017.

[7] DIAS, M. A. P. Logística, Transporte e Infraestrutura: armazenagem, operador logístico, gestão via TI, multimodal. São Paulo: Atlas, 2012.

[8] GOLDBARG, M. C.; LUNA, H. P. L. Otimização combinatória e programação linear. 2. ed. Rio de Janeiro: Elsevier Editora Ltda, 2005.

[9] GOMES JÚNIOR, A. C.; SOUZA, M. J. F.; MARTINS, A. X. Simulated Annealing aplicado à resolução do problema de roteamento de veículos com janela de tempo. Revista Transportes, v. 13, n. 2, 2005.

[10] HARA, C. M. Logística: armazenagem, distribuição, trade marketing. 4. ed. Campinas: Alínea Editora, 2011.

[11] HILliER, F. S.; LIEBERMAN, G. J. Introdução à Pesquisa Operacional. 8. ed. São Paulo: Mc Graw-Hill, 2006.

[12] KIRKPATRICK, S.; GELATT, C. D.; Vecchi, M. P. Optimization by Simulated Annealing. Science, v. 220, n. 4598, p. 671-680, 1983.

[13] KRAMER, R. H. F. R.; SUBRAMANIAN, A.; PENNA, P. H. V. Problema de roteamento de veículos assimétrico com frota heterogênea limitada: um estudo de 
caso em uma indústria de bebidas. Gestão da Produção, São Carlos, v. 23, n. 1, p. 165-176, mar. 2016.

[14] LEITE, L. O., GOMES JÚNIOR, A. C. Problema de Roteamento de Veículos com Coleta e Entrega Simultâneas: Um Estudo de Caso em uma Indústria de Alimentos localizada na região do Vale do Aço. In: EMEPRO, XIII, 2017, Juiz de Fora. Anais. Juiz de Fora, 2017.

[15] RAGSDAlE, C. T. Modelagem e Análise de Decisão. São Paulo: Cengage Learning, 2010 .

[16] ROSA, R. A. Gestão de Operações e Logística I. Florianópolis: Pnap Universidade Aberta, 2011.

[17] SANTANA, G. O. Fatores Competitivos dentro da Logística Empresarial. Administradores, out. 2016. Disponível em: <http://www.administradores.com.br/ artigos/academico/fatores-competitivos-dentro-da-logistica-empresarial/98848/>. Aces-so em: 07 dez, 2018.

[18] SOUZA, Marcone Jamilson Freitas. Inteligência computacional para otimização. DECOM, UFOP, 2011. Disponível em: <http://www.decom.ufop.br/prof/ marcone/Disciplinas/InteligenciaComputacional/InteligenciaComputacional.pdf $>$. Aces-so em: $07 \mathrm{dez}, 2018$. 